\title{
Discussions with Leaders: A Conversation Between Martin Pomper and Peter Choyke
}

\author{
Martin G. Pomper ${ }^{1}$ and Peter L. Choyke ${ }^{2}$ \\ ${ }^{1}$ Johns Hopkins Medical Institutions, Baltimore, Maryland; and ${ }^{2}$ National Cancer Institute, Bethesda, Maryland
}

Med ohannes Czernin, MD, editor in chief of The Journal of Nuclear Medicine, initiated in 2019 a series of recorded discussions with leaders in nuclear medicine and molecular imaging. This month he asked Peter L. Choyke, MD, Senior Investigator in the Molecular Imaging Program within the Center for Cancer Research at the National Cancer Institute (NCI; Bethesda, MD), to talk with Martin G. Pomper, $\mathrm{MD}, \mathrm{PhD}$, a professor in the Department of Radiology and Radiologic Science and director of Nuclear Medicine and Molecular Imaging at Johns Hopkins Medicine (Baltimore, MD). He is internationally known for development of new radiopharmaceuticals, including imaging and therapeutic agents targeting the prostate-specific membrane antigen (PSMA). He has numerous patents related to medical imaging, many of which have been licensed, as well as a number of imaging and theranostic agents in clinical trials.

Dr. Choyke: It is a great pleasure to talk with you. I would like to begin by asking about your backstory and how you got to where you are today. Could you describe your path?

Dr. Pomper: I grew up in north suburban Chicago, a product of public education and the space race, which was the rage when I was a child. I attended the University of Illinois at Urbana-Champaign, studied biochemistry as an undergraduate and then organic chemistry in graduate school as part the Medical Scholars Program, a progressive $\mathrm{MD} / \mathrm{PhD}$ program that had been recently established at the U of I. I was fortunate to have tremendous mentors, like John Katzenellenbogen, my graduate research advisor, who inspired me to apply chemistry to important biomedical problems. It just so happened that he was working with Michael Welch and some of the most exciting work they were doing involved PET radiopharmaceutical development. So I pivoted my graduate work toward imaging. After Illinois I went to Johns Hopkins as an intern on the Osler Medical Service. I stayed and did residencies in diagnostic radiology and nuclear medicine and a fellowship in neuroradiology. I have remained on the faculty since 1995.

Dr. Choyke: So how did you transition from neuroradiologist to molecular imager and chief of nuclear medicine?

Dr. Pomper: That was not a completely linear transition. When I was a radiology resident I knew that I was going to do academic medicine with a strong basic science component that leveraged my training in chemistry. Nevertheless, I always felt it was important to be in the clinic and be a physician first and foremost. I found that neuroradiology was extremely helpful to the neurosurgeons and neurologists and thought that would be a good clinical base. I started looking at PET neuroimaging, which has a strong history at Johns

COPYRIGHT @ 2020 by the Society of Nuclear Medicine and Molecular Imaging.
Hopkins. So even though I was a neuroradiology fellow, became a neuroradiology attending physician, and was a neuroradiologist for $20 \mathrm{y}$, the lion's share of my research was actually in trying to develop PET imaging agents for the brain and, a little later, for cancer. It was very natural when the position for director of the Division of Nuclear Medicine opened up in 2015 that I finally try to combine my research with a clinical leadership position. I have to say that the transition has not

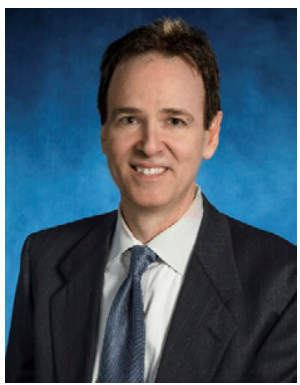

Martin G. Pomper, MD, PhD been trivial-to go from $20 \mathrm{y}$ in my clinical comfort zone to something new. We are fortunate to have a very strong and capable team that provides me with immense support.

Dr. Choyke: You have had some great discoveries in your career, but my favorite is your discovery of PSMA ligands to target prostate cancer. Could you tell that story?

Dr. Pomper: It started with an article by Paul Jackson I saw in the Journal of Medicinal Chemistry in 1996 (J Med Chem. 1996;39[2]:619-622). Back in those days I would find targets for imaging by looking at the Journal of Medicinal Chemistry or Cancer Research. One that caught my eye was about high-affinity inhibitors for a brain enzyme that generates glutamate, called $N$-acetylated $\alpha$-linked acidic dipeptidase (NAALADase). In graduate school I put together a proposal for a departmental seminar on imaging glutamatergic neurotransmission but got sidetracked and did not pursue it at the time. When I saw the Jackson paper I thought I could functionalize his inhibitors for PET so we could get a handle on what was happening with at least a portion of the neurotransmitter pool of glutamate. However, when I looked at the compounds, they were very hydrophilic and probably would not get into the brain easily. So I read a little further about NAALADase and saw a concurrent paper by Ruth Carter, in which they showed that the brain enzyme was homologous to PSMA (Proc Natl Acad Science USA. 1996:23;93[2]:749-753). I immediately thought we could use Jackson's compounds to image prostate cancer, which did not have the problem of the blood-brain barrier and needed better imaging. I submitted a proposal to the Department of Defense, but that was not funded. I had a tritium-labeled version made, but never tested it because I got too busy in the clinic. Things did not take off until 1999, when I met Alan Kozikowski, a talented chemist then at Georgetown University (DC), who was a guest lecturer for pharmacology at Johns Hopkins. I described to Alan how we were working on NAALADase inhibitors for targeting prostate cancer, and he 
mentioned that he was developing what he called NAAG peptidase (same as NAALADase) inhibitors as neuroprotective agents. After discussing his portfolio, he provided a precursor that could be radiolabeled with ${ }^{11} \mathrm{C}$. These were the urea compounds that are now very familiar. An initial study in rodents and a nonhuman primate using the kidney as a surrogate for a PSMA-expressing tumor demonstrated specific binding (admittedly, somewhat to my surprise). In the next publication, in 2005, we used a PSMA-expressing model of prostate cancer, and after that we started making analogs for a variety of isotopes and modalities. Probably the next most important discovery was a few years later: through computational docking we found that we needed about $20 \AA$ between the urea and bulky substituents if we wanted to attach chelators or nanoparticles or make fluorescent agents. My colleague, Sangeeta Ray, made the first radiometalated analogs, and they worked well. We have been making derivatives ever since.

Dr. Choyke: It is an amazing story. One of the reasons it was so well adapted to prostate cancer is that there is a serum biomarker for prostate cancer, prostate-specific antigen (PSA). In the setting of recurrent tumor, you have a very sensitive blood test that selects patients for imaging. Not many other cancers have that advantage. As we look forward to developing additional agents that would work on other cancers, how important is it that we work with the serum biomarker community? lifting, which begins just after the toxicology studies, which can also be hard for an academic lab to handle. Patent protection of intellectual property is generally necessary to motivate commercial entities to come in for the phase 2 and pivotal trials. NIH grants rarely cover beyond phase 1 . The process has not been done systematically in the past, when scientists understandably focused on their favorite compound or the next bigger, better agent, without systematic testing of what we have already. But the field is maturing, a roadmap is emerging, and more collaborations and consortia are partnering to standardize protocols and bring a few promising agents to the clinic. The process is accelerating, with several New Drug Applications coming online within the next couple of years.

The gallium-labeled somatostatin receptor radiotracers provide an excellent example. There was no good way to treat patients with metastatic neuroendocrine tumors, so the need for the theranostic concept (imaging to select patients to treat with essentially the same molecule) was ready to be tested in earnest-by a commercial entity with an eye to FDA approval with reimbursement. The NETTER-1 trial showed how valuable theranostics could be. Prostate cancer is a little bit different, because there are other therapies, and PSMA-targeted radiotherapies will either find a niche or be used in combination with other treatments in the future. I have seen firsthand how these therapies dramatically improve patients' quality of life as well as the lack of side effects that

\section{"I think as a nation we could add a zero onto the current NIH budget of $\$ 39$ billion, but this is a matter of national priorities."}

Dr. Pomper: It is absolutely critical that we do that for a couple of reasons. PSA has its pluses and minuses, but it has been a boon to imaging because we can use PSA changes to trigger imaging. You can also use PSMA imaging to guide and monitor treatments like stereotactic radiation. More recently, with molecular radiotherapeutics, we can use PSA as a response marker. Imaging studies are much more expensive than serum markers, but imaging does 2 things that you cannot get from a serum or urine biomarker. First, you get spatial localization, and, second, the imaging biomarker is in an undiluted form in its native environment. I could foresee using an inexpensive screening test, such as circulating tumor DNA, where you need about 50 million cells to be able to detect the tumor. If you get a hit, you can then scan the patient and require on the order of only 15 million cells in a metastasis to visualize on a PET scan. So we are using both liquid biopsy and imaging now. The former is a screen, and the latter provides spatial information that enables tailoring of management.

Dr. Choyke: Totally agree. The 2 best examples of low-molecularweight imaging agents are the somatostatin-targeted and the PSMAtargeted agents. But both have taken an awful long time to come to fruition, and, in the United States, PSMA really has not come to fruition. So how can we accelerate this process?

Dr. Pomper: By linking closer to therapy and patient management-and starting off with well-designed, prospective trials that can be used for registration of the compound. Imaging can facilitate therapy, and it is not difficult to bring imaging agents to the clinic if you are working with high specific activity, as with PET tracers. The key is to work on a compelling biologic target that addresses an unmet need so that professional societies and foundations will be willing to provide domain expertise and cover the financial heavy we see compared with standard chemotherapy. As we acquire more experience with these agents, medical oncologists will recognize them as valuable adjuncts to their work. As long as we partner with them closely, this collaboration will hasten the acceptance of more of these agents going forward.

Dr. Choyke: So that segues nicely to the next question about targeted radionuclide therapy. Who is going to be doing this in the United States?

Dr. Pomper: We have a different model for nuclear medicine in the United States, where it is generally a section within a radiology department, except, of course, for Dr. Czernin, who has the European model at the University of California Los Angeles. His is really a fantastic, nimble program, and I am very envious of it. Nevertheless, in the United States the model has been for nuclear medicine physicians to administer radiotherapeutics. In most places across the country, nuclear medicine is hoping to make this part of their bread and butter and is opening up centers to use the first-generation agents. The main questions to answer are: who is going to take the best care of the patients and who is going to partner most closely with the referral base? Nuclear medicine physicians, radiologists, and radiation oncologists are all obviously capable of taking care of patients. This is actually an opportunity for radiology and nuclear medicine, which have traditionally not been tremendously patient-facing, to revisit this and try to lead in this area. If we work very closely with medical oncologists, then we can lead, because these agents emanated from nuclear medicine and we know them best. The answer is always what is in the best interest of the patients. It is not trivial to deliver these molecular radiotherapeutics. Even though it sounds like a standard infusion, it is different from infusing chemotherapy. We have technologists 
who are very specifically trained to do this kind of thing. It is definitely an operation that needs tender loving care on all levels.

Dr. Choyke: Let me switch gears. I know you have put a lot of thought into the future of nuclear medicine and nuclear medicine research. You have created a highly innovative cross-disciplinary environment at Johns Hopkins, but I know that research funding is increasingly competitive and institutions have increasing pressures on them to generate clinical income. But as someone who has done this even in a challenging environment, what do you think we need to do as a nation to get back on track?

Dr. Pomper: Wow. . . as a nation! The NIH budget in real terms has been declining, but in some areas funding is improving. Paylines are at or near the 20th percentile for new investigators doing research related to Alzheimer disease, for example. But the NCI payline is currently only at $8 \%$. I think as a nation we could add a zero onto the current $\mathrm{NIH}$ budget of $\$ 39$ billion, but this is a matter of national priorities. The NIH is the absolute jewel of biomedical research in the world; there is nothing quite like it anywhere else. For all the complaining that we do about it, it is a merit-based system. It is remarkable that the federal government has the foresight to keep it going. It should be better resourced, because most of the great ideas that go on to become commercial products arise from discoveries made in academic labs. The research happens in academia, and development occurs in the pharmaceutical and device industries. Research is being outsourced increasingly to academic centers. I think we need to make it very clear to our leaders that the NIH is important-more important than it is currently being resourced.

Dr. Choyke: If you look at a map of activity in academic medical centers in the United States, sometimes it seems like the number of lights still on decreases every day. It is very frustrating to watch. How do you keep going?

Dr. Pomper: We are seeing academic hospitals encountering increasing difficulty staying solvent and less able to fund the academic mission. That is, in part, because health care costs in the United States are unsustainable, so academic hospitals (which tend to be less efficient), as well as the fee-for-service system, are being disrupted. The entire culture of managing care in the United States has to change so that we focus on keeping people healthy from day 1 . So, for example, we should see more emphasis on primary prevention and as much, if not more, emphasis on diagnostics relative to therapeutics. As these changes take hold, academic physicians and researchers will have to find creative solutions for supplementing their funding other than through their institutions and $\mathrm{NIH}$, for the reasons discussed above. As academic hospitals become more aligned, from the dean's or CEO's office all the way down to the departmental section chiefs, the departments do not have the kind of discretion to invest directly in research that they had in the past-particularly radiology departments, which have historically been profit centers. All of this has put more pressure on physician/scientists and on the PhD scientists in clinical departments. We need innovation, entrepreneurship, and academic-industry partnerships, the latter managed gingerly. So we are thinking about new models for funding our mission, which involve more of these nontraditional areas. The constant challenges and knowing that if we are successful we will have even wider impact on our patients through some of these routes is what keeps me going, personally.

Dr. Choyke: You have been very generous with your time, and I want to ask a final question. If a young Marty Pomper came into your office today seeking advice, what would you tell him?

Dr. Pomper: When I was an undergraduate student I approached John Katzenellenbogen about working in his lab. Sizing me up as knowing little to nothing about chemistry, he nevertheless would meet with me every couple of weeks to discuss "cancer," in general, because I told him I wanted to help cure it through chemistry. By taking that interest in my career, devoting his precious time, I felt valued. I try to show similar value to motivated young people and want to know what they are thinking. I particularly enjoy when they come to me with ideas they would like to pursue. I then try to advise or resource them, if I can, after a little vetting of the idea. The key is, particularly for new and likely overwhelmed clinician/ scientists, that they see a path to success and that there are more senior people around who understand them and will help them get there. 